\title{
De la competencia intercultural en la adquisición de una segunda lengua o lengua extranjera: conceptos, metodología y revisión de métodos
}

\author{
Ana M. Rico Martín \\ Dpto. de Didáctica de la Lengua y la Literatura \\ Facultad de Educación y Humanidades de Melilla \\ Universidad de Granada
}

Recibido: 24 abril 2004 / Versión aceptada: 28 mayo 2004

\begin{abstract}
RESUMEN: En este artículo se analizan diferentes cuestiones en torno a la competencia intercultural de una lengua extranjera: la importancia que tiene en la adquisición y el uso de esa lengua; la evolución de los objetivos finales de la enseñanza de lenguas: desde los conocimientos lingüísticos a la competencia intercultural; los aspectos socioculturales del área de Lenguas Extranjeras en el actual sistema educativo español y la metodología más apropiada para su enseñanza donde se incluyen algunas técnicas para trabajarla en el aula.

Además el artículo presenta un breve estudio comparativo del componente sociocultural de los principales enfoques y métodos de enseñanza de lenguas extranjeras del siglo XX.
\end{abstract}

Palabras clave: enseñanza de lenguas extranjeras, dimensión sociocultural, competencia intercultural.

\begin{abstract}
In this paper different questions about the intercultural competence of a foreign language are analysed: its importance in that language acquisition and use; the evolution of central objectives in language education: from linguistic knowledge to intercultural competence; sociocultural aspects in the area of Foreign Language in the Spanish educational system and the most appropriate methodology to the teaching of intercultural competence where some techniques to work in the classroom are included. Besides the article presents a brief comparative study of the sociocultural dimension in the main methods of foreign language teaching in the 20th century.

Key words: foreign language teaching, sociocultural aspects, intercultural competence.
\end{abstract}

\section{INTRODUCCIÓN: DESDE LA COMPETENCIA LINGÜÍSTICA A LA COMPETENCIA INTERCULTURAL}

Cuando hablamos de educación intercultural solemos relacionarla con la situación de los inmigrantes o "nuevos ciudadanos" en el país de acogida. La mayor parte de los textos que trabajan la intercultura se refieren a la prevención y modificación de los prejuicios y estereotipos negativos hacia los otros y al desarrollo de actitudes que favorezcan la convivencia 
más que multicultural, intercultural ${ }^{1}$, aunque se haya polemizado mucho sobre si es factible esa interculturalidad o si se trata de una utopía y sólo nos podemos quedar en la mera coexistencia de diferentes culturas (multiculturalidad). En uno u otro caso, siempre se trabajará para abrir el camino de la integración. Graziella Favaro (21) señala tres palabras clave a la hora de crear una "scuola di tutti i bambini", autóctonos e inmigrantes, éstas son: "l'accoglienza, l'educazione lingüística, l'educazione interculturale". La autora se refiere a la educación lingüística como un proyecto que debe tener todo centro que acoja a los "nuevos ciudadanos" y que debe estar en continua exploración e innovación metodológica y didáctica al tener la lengua nacional -la autora alude al italiano- el carácter de L2 (segunda lengua) ante la inmigración de adultos y niños; se reconoce así mismo el papel tan importante que tiene la lengua y la comunicación en la integración del individuo.

En España, por ejemplo, la competencia intercultural, objeto de nuestro trabajo, aparece al mismo tiempo que proliferan los estudios sobre educación intercultural.

Relacionada estrechamente con esta educación lingüística señalada por Favaro, queremos referirnos a la competencia intercultural en la adquisición de una L2 o de una lengua extranjera, mucho más fácil de desarrollar en el primer caso pues el hecho de vivir en el país de la lengua de estudio proporciona una serie de experiencias y recursos del "aquí y ahora" difíciles de conseguir cuando se estudia una lengua extranjera descontextualizada.

No nos extrañe plantearnos que nuestros alumnos alcancen esa competencia intercultural, además de la lingüística, si consideramos que casi el $10 \%$ de la población de la Unión Europea está constituido por ciudadanos de países diferentes de aquél donde está residiendo; por otro lado, cerca del $50 \%$ de los alumnos está diariamente en contacto con personas que hablan una lengua diferente a la suya. Un dato más: prácticamente todos los países de la Unión Europea son, sobre todo a partir de la década de los 80, países de inmigración (Surian, 2000:43).

Ya a partir de los 70, con la fuerza de los enfoques comunicativos como método imperante en la enseñanza de idiomas, muchos autores coincidieron en que hacía falta algo más que conocimiento lingüístico para poder dominar una nueva lengua en cualquier situación real, Van Ek (1984) es quizás el que presenta un modelo más detallado de esas otras competencias que han de ser adquiridas complementariamente para propósitos comunicativos:

- Competencia lingüística o capacidad de conocer y usar la lengua correctamente atendiendo a todos sus componentes: fonológico, morfológico, sintáctico, semántico y léxico.

- Competencia sociolingüística o capacidad de comunicarse con precisión en contextos diferentes, con personas diferentes y cuando hay diferentes intenciones en la comunicación.

${ }^{1}$ Al respecto hay editado un libro del Grupo de Investigación "Innovación Curricular en Contextos Multiculturales" al que pertenece la autora del artículo: Sánchez Fernández, S. y Mesa Franco, M. C. (coords.) (2002). Los relatos de convivencia como recurso didáctico. Elaboración de materiales curriculares como estrategia para la prevención y modificación de estereotipos negativos en contextos multiculturales. Málaga: Aljibe. En él, tras una investigación concienzuda sobre los relatos de vida de los principales grupos culturales de Melilla, se recoge una serie de actividades con el propósito que refleja el título. 
- Competencia discursiva o capacidad de emplear estrategias apropiadas para construir e interpretar textos diferentes.

- Competencia estratégica o capacidad de aplicar recursos de comunicación verbal y no verbal para llenar los vacíos en el saber lingüístico del hablante.

- Competencia sociocultural o conciencia del contexto sociocultural en el que es empleada la lengua por los hablantes nativos y de las formas en que este contexto afecta a la selección y el efecto comunicativo de determinadas formas de la lengua.

- Competencia social o capacidad de tomar la iniciativa en una conversación, por ejemplo, saber cuándo interrumpir a alguien que habla con grosería; es decir, en un concepto más amplio, conocer las convenciones sociales del grupo.

A menudo y desde hace mucho tiempo, el profesor de lengua, en general, de lengua extranjera, en particular, ha centrado su labor en el contenido lingüístico dejando a un lado los factores sociológicos y culturales que intervienen en todo uso de la lengua. Se trataba entonces de enseñar formas lingüísticas para hablar y escribir con corrección, sin faltar tampoco a la coherencia ni a la cohesión, pero la adecuación se hacía de cara a un hipotético y restringido receptor del mensaje, poniendo en peligro, quizás, el éxito comunicativo en una situación real. Hoy día entendemos que los receptores son múltiples y muy diferentes, además de que la finalidad de nuestra producción lingüística, los usos y contextos han pasado a primer plano en la enseñanza-aprendizaje de la lengua.

Está claro que no basta con el desarrollo de una buena competencia lingüística si ésta no se haya enmarcada en una competencia comunicativa ${ }^{2}$ mucho más amplia, donde se incluiría nuestra competencia intercultural. Recibir un input meramente lingüístico no ayuda a la total y efectiva descodificación del mensaje, bien que, si nos movemos en el campo de las actitudes y la metacognición, el alumno puede ser capaz de comprender un mensaje aunque no comprenda todos sus elementos lingüísticos, en ello juega un gran papel sus experiencias y estrategias de uso de la primera lengua (L1).

Evidentemente, el aprendiz de una nueva lengua debe dar mayor importancia al mensaje en su globalidad que a la forma del mismo. Estaríamos, pues, ante un proceso de adquisición de la lengua extranjera más allá del aprendizaje, normalmente lingüístico, que caracteriza a la primera etapa en la enseñanza de esa lengua.

Pero ¿cómo describiríamos la competencia intercultural? De la definición de competencia cultural que hacen Hains, Lynch y Winton (1997:2) surge la referida a la que nos compete: habilidad que tienen para relacionarse y comunicarse de manera efectiva los individuos que no comparten la misma cultura, etnicidad, idioma u otras variables importantes.

Según Meyer (1991), más explícito, "la competencia intercultural, como parte de una amplia competencia del hablante de una lengua extranjera, identifica la habilidad de una persona de actuar de forma adecuada y flexible al enfrentarse con acciones, actitudes y expectativas de personas de otras culturas". Incluiría, además, "la capacidad de estabilizar la propia identidad en el proceso de mediación entre culturas y la de ayudar a otras personas a estabilizar la suya" (en Oliveras, 2000:38).

\footnotetext{
${ }^{2}$ Obviamos en estas líneas toda la literatura existente sobre la competencia y la actuación lingüísticas de Chomsky y la introducción del concepto de competencia comunicativa por parte de Hymes y otros en el campo de la enseñanza de idiomas.
} 
Por otro lado, Vilà (2002), partiendo del modelo de competencia comunicativa intercultural desarrollado por Chen (1998), entiende que esta competencia debe comprender tres tipos de habilidades:

- Habilidades cognitivas, referidas al conocimiento y la conciencia de elementos comunicativos y culturales de la propia cultura y de otras.

- Habilidades afectivas o capacidades de emitir respuestas emocionales positivas y de controlar las negativas.

- Habilidades comportamentales, son habilidades verbales y no verbales que evidencian una adaptación de la conducta a la situación y al contexto.

Vistas estas consideraciones, estaremos de acuerdo en que en este complejo concepto de competencia intercultural intervienen amplias disciplinas y ramas científicas tales como la psicología, la lingüística, la sociolingüística, la antropología, la filosofía y la etnografía. Todas ellas con mucho que decir e investigar a propósito de esta competencia.

\section{La COMPETENCIA INTERCULTURAL EN LA ADQUiSición DE UNA LENGUa EXTRANJERA O UNA L2}

Es sobre todo a partir del ámbito empresarial desde el que se han iniciado diferentes estudios sobre las necesidades de la comunicación intercultural (Hall, 1978, 1986, 1989; Hofstede, 1984; Baliga y Barker, 1985; o Brewster, 1991, entre otros), ello ha tenido como consecuencia el desarrollo de múltiples ofertas en formación cultural con el objetivo final de ayudar a entender la psicología y el comportamiento de personas de otras sociedades evitando así problemas y malentendidos condicionados culturalmente. Los destinatarios son muy variados: hombres de negocios que planean una fusión internacional o que ya han comenzado con ella, diplomáticos y personal de consulados y embajadas, personas que quieren ampliar el campo de las relaciones públicas más allá de los límites nacionales, o funcionarios y empleados que trabajan con inmigrantes y extranjeros en España.

En el ámbito del aprendizaje de lenguas extranjeras los objetivos que se pretenden alcanzar son:

- Despertar y desarrollar la sensibilidad cultural.

- Comprender mejor las formas de pensar, los presupuestos, los valores y el mundo emocional de la cultura de otro país.

- Reconocer las diferentes dimensiones en las que están organizadas las otras culturas como la forma de concebir el tiempo y el espacio o las prioridades en tareas y relaciones.

- Reconocer malentendidos y desenvolverse mejor en situaciones parecidas del futuro. 
En la enseñanza reglada española, por ejemplo, si revisamos el nuevo currículum de la L.O.C.E. ${ }^{3}$ para el área de Lengua Extranjera de la Educación Primaria se reconoce en el aprendizaje de esta lengua la importancia de "la comprensión de costumbres y formas de vida diferentes, la tolerancia y el respeto entre los países y sus hablantes" (B.O.E. n. ${ }^{\circ}$ 157, 2003:25460), para ello uno de los ejes vertebrales en torno a los que se organizan los contenidos de enseñanza son los aspectos socioculturales que introducen nociones como "costumbres, tradiciones, formas de organización y de relación personal y social al tiempo que se favorece el esfuerzo y la confianza en la propia capacidad para aprender la lengua extranjera" (B.O.E. n. $\left.{ }^{\circ} 157,2003: 25460\right)$.

No obstante, la nueva ley parte de la premisa del Consejo de Europa que señala como objetivo último del aprendizaje de una lengua extranjera que los alumnos alcancen progresivamente grados de competencia lingüística.

Entre los contenidos del eje al que nos hemos referidos encontramos sobre todo la creación y desarrollo de determinadas actitudes (B.O.E. n. ${ }^{\circ}$ 157, 2003:25460-62):

- Curiosidad, interés y respeto por otra lengua y cultura distinta a la propia.

- Actitud abierta hacia las personas que hablan la lengua extranjera.

- Valoración de nuestra propia cultura.

- Reconocimiento de similitudes y diferencias entre aspectos de la vida cotidiana en los países donde se habla la lengua extranjera y los propios.

- Interés por relacionarse con hablantes de la lengua extranjera, estableciendo contactos personales a través de los medios que nos proporcionan las tecnologías.

En la etapa de la Educación Secundaria Obligatoria se continúa la enseñanza de la Primaria pero se hace mayor insistencia en el desarrollo de la competencia comunicativa de los alumnos, por ejemplo, se trabajan tareas de comunicación que deben estar "enmarcadas en ámbitos que pueden ser de tipo público (todo lo relacionado con la interacción social cotidiana), personal (relaciones familiares y prácticas sociales individuales), laboral o educativo" (B.O.E. n. ${ }^{\circ}$ 157, 2003:25695), desarrollando a su vez la subcompetencia sociolingüística (bloque tercero de los contenidos de enseñanza), con los mismos aspectos trabajados en Primaria pero enfocados más hacia la intercultura.

Como comprobamos, estos contenidos son más explícitos que los que reflejaba la anterior ley de educación, L.O.G.S.E., subsanando en lo posible lo que algunos estudiosos de la materia señalaron como visión estática y simplista de la cultura extranjera (Trujillo, 2001). Con la nueva ley, partiendo de los primeros ciclos de Primaria donde se trabaja una competencia sociocultural ante todo, se tiende a desembocar en una competencia intercultural en la Educación Secundaria que implica al aprendiz de una nueva lengua tanto en aspectos cognitivos como actitudinales, insistiendo en estos últimos.

${ }^{3}$ La L.O.C.E. es la nueva Ley Orgánica 10/2002, de 23 de diciembre, de Calidad de la Educación (B.O.E. n. $307:$ 45188-45220) que actualmente está siendo modificada en algunos de sus aspectos. Nos hallamos en un periodo de transición entre la L.O.G.S.E. y la L.O.C.E., se van introduciendo algunas innovaciones de la segunda ley que en ningún momento vendría a derogar totalmente la anterior. 
Cuestión aparte es cuando Meyer (1991) ${ }^{4}$ diferencia tres estadios en el desarrollo de la competencia intercultural refiriéndose siempre al estado mental y actitudinal del aprendiz de la lengua extranjera, más allá de la adquisición de la competencia lingüística:

- Nivel monocultural, donde el alumno se mantiene siempre bajo el punto de vista de su propia cultura, todo lo interpreta según las reglas de la misma.

- Nivel intercultural, se halla ya entre ambas culturas, la propia y aquella que es objeto de estudio, las compara y es capaz de establecer semejanzas y diferencias.

- Nivel transcultural, cuando se encuentra por encima de ambas culturas y actúa de mediador de ellas con los principios fundamentales de cooperación y comunicación.

El docente de una lengua extranjera debe pretender que sus alumnos adquieran el último nivel que corresponde al alcanzado por bilingües equilibrados (equilingües), quienes dominan igualmente la lengua materna y la L2 o extranjera.

Refiriéndonos en estos momentos a la metodología de trabajo, conviene enfocarla a partir de métodos dialógicos, experienciales y de investigación, donde se fomenta la participación y el espíritu de cooperación de los alumnos.

Las pautas generales para trabajar según esta metodología, por ejemplo con un enfoque socioafectivo, serían las siguientes:

1. Vivenciar una experiencia donde se manifieste la diversidad de puntos de vistas de dos culturas, ya sea real o simulada, por parte del alumno como miembro de su grupo o de un grupo menor dentro del gran grupo de clase. Aquí tendrían cabida diferentes situaciones de simulación, juegos de roles...

2. Describir y analizar esa experiencia, no sólo el transcurso de la misma y la resolución del posible conflicto planteado, sino, sobre todo, las reacciones emocionales, las variaciones de los comportamientos y de las actitudes de los individuos-alumnos implicados.

3. Contrastar la experiencia vivida con situaciones de la vida real. Esto es muy importante pues permite al alumno valorar la actividad grupal como un ejercicio efectivo fuera del aula escolar, más allá del mero contexto educativo (4. ${ }^{\circ}$ punto).

4. Evaluar la actividad. Como docentes nos interesa conocer qué grado de aceptación y de éxito tiene, podemos hacerlo a través de un pequeño coloquio para garantizar la espontaneidad y la libre expresión de los alumnos, con lo que conoceremos sus opiniones más sinceras.

Fuera de la metodología socioafectiva, pues conlleva un trabajo más personal e individualizado, pero que favorece asimismo la adquisición de la competencia intercultural está la realización de cuestionarios sobre autoanálisis cultural y las técnicas de sugestopedia ${ }^{5}$

${ }^{4}$ Op. Cit. nota 3.

${ }^{5}$ Relacionado con el yoga y la psicología soviética, este método creado por el educador y psiquiatra búlgaro Georgi Lozanov trata de controlar las influencias irracionales e inconscientes a las que los seres humanos estamos constantemente respondiendo y reconducirlas para mejorar el aprendizaje de una lengua extranjera. Ha sido un método muy criticado por su falta de rigor científico pero halagado, a su vez, por la efectividad de sus técnicas. Volveremos a él unas páginas más adelante. 
(recomendadas sobre todo por distintos centros de desarrollo personal entre cuyos objetivos destaca la competencia intercultural de una lengua no materna).

Trabajando de otro modo, cuando la inmersión en la propia sociedad de la lengua extranjera -en este caso L2 - no es posible, la competencia intercultural puede lograrse, entre otros recursos, a través de una serie de textos, orales y escritos, con determinadas cualidades para que su aprovechamiento sea óptimo en la clase de lengua extranjera (Cambra, 1989:6568):

- Autenticidad o realismo. Intentaremos no trabajar con textos simplificados ni adaptados que pequen de distorsionados, demasiado explícitos o faltos de contenido por darle mayor importancia a su forma.

- Legibilidad. En el caso de un texto escrito, es importante que éste favorezca una lectura fluida además de eficaz, atendiendo a los aspectos visuales y mentales de la percepción, su tipografía, iconicidad, estructura y redundancia.

- Diversidad. Pareja ésta a la que encontramos en todas las manifestaciones lingüísticas de nuestra vida cotidiana.

- Cohesión y coherencia. Las cuales facilitarán la aprehensión del sentido del texto mediante elementos que mantengan la referencia: preposiciones, conectores lógicotemporales, palabras clave... siempre haciendo representativo al texto del uso normal de la lengua.

Cambra incluye un cuadro de J.M. Adam (1985) donde se recogen ocho tipos de textos según el acto de discurso que se quiera trabajar, los ejemplos textuales podrán adaptarse a la edad, necesidades y condiciones de los alumnos e, indudablemente, hay unos más apropiados

\begin{tabular}{|l|c|l|}
\hline \multicolumn{1}{|c|}{ ACTE DISCOURS } & TYPE TEXTUEL & \multicolumn{1}{c|}{ EXEMPLES } \\
\hline $\begin{array}{l}\text { ASSERTER } \\
\text { (énoncés de faire) }\end{array}$ & NARRATIF & $\begin{array}{l}\text { Reportage } \\
\text { Conte } \\
\text { B.D. }\end{array}$ \\
\hline $\begin{array}{l}\text { ASSERTER } \\
\text { (énoncés d'état) }\end{array}$ & DESCRIPTIF & $\begin{array}{l}\text { Dictionnaire unilingue } \\
\text { Programme d'émissions } \\
\text { T.V. }\end{array}$ \\
\hline $\begin{array}{l}\text { EXPLIQUER OU FAIRE } \\
\text { COMPRENDRE }\end{array}$ & EXPLICATIF & $\begin{array}{l}\text { Documentaires } \\
\text { Livres de texte }\end{array}$ \\
\hline CONVAINCRE & ARGUMENTATIF & Texte publicitaire \\
\hline $\begin{array}{l}\text { ORDONNER } \\
\text { INJONCTIF }\end{array}$ & $\begin{array}{l}\text { Règles de jeux } \\
\text { Modes d'emploi } \\
\text { Notices montage } \\
\text { Recettes cuisine }\end{array}$ \\
\hline $\begin{array}{l}\text { QREDIRE poétique, } \\
\text { PROMETTRE } \\
\text { ANNONCER } \\
\text { APPELER } \\
\text { MENACER... }\end{array}$ & PREDICTIF & Rulletin météo \\
\hline $\begin{array}{l}\text { (fonction } \\
\text { Jakobson) }\end{array}$ & RHETORIQUE & $\begin{array}{l}\text { Interview } \\
\text { Dialogue théâtral } \\
\text { Coème } \\
\text { Dicton } \\
\text { Slogan }\end{array}$ \\
\hline
\end{tabular}

Cuadro de Cambra (1989: 67) 
que otros para trabajar los aspectos socioculturales de la nueva lengua y desarrollar lo que esta misma autora señala como competencias referencial (en los dominios de la experiencia y el conocimiento), relacional (en la regulación de los intercambios interpersonales) y situacional (de los contextos de según las opciones comunicativas elegidas) dentro de la gran competencia comunicativa (Cambra 1989:70-71).

Hay que tener cuidado con estos tipos textuales pues debemos considerarlos como conjuntos de rasgos que no siempre se corresponden con los actos que realizan (preguntar, afirmar o exclamar). Como clasificación general nos vale ésta de Adam, pero en un texto real interactúan diversos tipos de textos que muchas veces se encubren, por ejemplo, una cuestión no siempre busca información sino que puede tener un matiz claramente exhortativo: ¿me das tu libro?

El verdadero enriquecimiento lingüístico-comunicativo vendrá no por el análisis de determinados textos sino por las redes y asociaciones que establecemos entre ellos, yendo más allá de la mera forma textual.

Richards y Rogers (1998:32) señalan, por ejemplo, que los materiales de enseñanza útiles en una metodología funcional comunicativa, de las más cercanas a nuestro propósito, deben centrarse en las habilidades comunicativas de interpretación, expresión y negociación, insistiendo mejor en los intercambios de información comprensible, relevantes e interesantes que en la presentación de la forma gramatical.

En la programación de lengua extranjera o L2 debe considerarse importante el trabajo con la conversación coloquial o cotidiana pues es el texto más usado cuando interactuamos socialmente.

Si bien este uso oral está bastante menoscabado en la enseñanza de la lengua materna, sí se trabaja en la de lenguas extranjeras, pero cuidado, es muy frecuente que este tipo de texto aparezca, ya con su forma oral ya transcrito, demasiado manipulado y alejado del habla habitual. Porter y Roberts (1981) señalan esta manipulación que aparece en múltiples aspectos más evidentes cuando se trata de diálogos grabados: entonación artificial, pronunciación estándar, repetición estructural, oraciones perfectas, cambios de turno demasiado claros, vocabulario limitado o referencias excesivamente explícitas.

Para trabajar una conversación debemos tener presentes las siguientes observaciones:

- Al conversar, quienes hablan se suceden en la toma de palabra, distribuyéndose por turnos. Esta distribución no está previamente establecida, como tampoco lo está la duración de la conversación.

- Entre un turno y el próximo, casi no hay pausas. Sin embargo los solapamientos no son habituales, y los que se producen son breves.

- Como hablantes, somos capaces de reconocer momentos apropiados para iniciar nuestro turno de habla.

(Unamuno, 2003:44)

Estos turnos están precedidos y cerrados por fórmulas de saludo y de despedida que, para su efectividad, deben ir acompañados de elementos o rasgos no lingüísticos que enmarcan el espacio conversacional como es, por ejemplo, pararse hablante frente a hablante y, dirigiendo la mirada hacia el rostro, iniciar la conversación, es lo que llamamos la interacción cara a cara.

Pero esta actividad conversacional tiene un riesgo: al trabajarla en parejas o en grupos con otros alumnos éstos en numerosas ocasiones no pueden ser buenos compañeros de 
conversación porque son incapaces de proporcionar información de forma correcta cuando se solicita.

Unamuno nos propone como actividad el crear en el aula una conversateca o archivo de conversaciones reales, espontáneas pero con consentimiento de los participantes, grabadas y acompañadas de fichas con datos contextuales como los que aparecen en la tabla siguiente. Cada grupo de alumnos proporcionará una o varias conversaciones, las transcribirá y rellenará una ficha parecida a la del cuadro con los datos de observación sobre el contexto. Se trata de sistematizar lo que hacemos cotidianamente.

\begin{tabular}{|c|c|c|}
\hline & & - Aula \\
\hline & - Calle & - Patio \\
\hline & - $\quad$ Escuela & - $\quad$ Comedor \\
\hline & & $\bullet \quad[\ldots]$ \\
\hline \multicolumn{3}{|l|}{ SITUACIÓN } \\
\hline & & - Comedor \\
\hline & - Tienda & - Cocina \\
\hline & - Casa & - Baño \\
\hline & & $\begin{array}{ll} & {[\ldots]} \\
\end{array}$ \\
\hline & $\cdot \quad[\ldots]$ & \\
\hline & & - $\quad$ Conocidos /desconocidos \\
\hline & 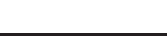 & - Jefe-empleado \\
\hline & - $\quad$ Relación & - $\quad$ Colegas \\
\hline & & - $\quad$ Padre-hijo \\
\hline & & - $\quad$ Hermanas \\
\hline & & $\begin{array}{ll} & {[\ldots]} \\
\end{array}$ \\
\hline & & • $\quad$ Niños \\
\hline PARTICIPANTES & - $\quad$ Edad & $\begin{array}{ll}\text { - } & \text { Adolescentes } \\
\end{array}$ \\
\hline & & - $\quad$ Adultos \\
\hline & & - $\quad[\ldots]$ \\
\hline & & \\
\hline & & - $\quad$ Región \\
\hline & & - País \\
\hline & - $\quad$ Origen & - $\quad$ Lengua \\
\hline & & - $\quad$ Grupo social \\
\hline & $\begin{array}{ll} & {[\ldots]}\end{array}$ & \\
\hline & \multirow{3}{*}{\multicolumn{2}{|c|}{$\begin{array}{ll}\text { - } & \text { Comprar-vender } \\
\text { - } & \text { Enseñar-aprender } \\
\text { - } & \text { Informar-pedir información } \\
\text { Pasar el rato }\end{array}$}} \\
\hline FINALIDADES & & \\
\hline & & \\
\hline
\end{tabular}

Cuadro adaptado de Unamuno (2003: 47). 
Una tarea parecida sería la elaboración de un diario donde el alumno de lengua extranjera recoja momentos de conversación en los que él considere que ha habido un malentendido y que lo explique.

En cualquier caso, las actividades útiles para el desarrollo de la competencia intercultural son muy variadas, podemos incluir en nuestra programación tareas que impliquen una investigación lingüística que motive la curiosidad de nuestros alumnos hacia otras lenguas y sus diferentes usos sociales, creando así una actitud positiva y abierta hacia el multilingüismo.

En este momento vienen al caso las palabras de Piaget cuando llamó al proceso de aprendizaje de la lengua "construir la realidad" y ello se logra mejor a través de experiencias de primera mano que generan una motivación alta e integradora que facilita, a su vez, el progreso más rápido en el aprendizaje de la lengua meta. Esto sólo es posible en la adquisición de una segunda lengua, cuando el alumno está inmerso en una situación directa o real de comunicación intercultural y se adapta a ese nuevo mundo con mayor o menor dificultad según su contexto cultural, su experiencia previa o su propia personalidad. En el caso de una lengua extranjera conviene reiterar experiencias del mismo tipo para conceder una alta credibilidad a expresiones lingüísticas usadas en el lenguaje común, lo que hace que tales expresiones sean retenidas en la memoria más fácilmente.

En cualquier caso, las propuestas didácticas deben encaminarse a la familiarización del alumno con métodos que combinen la participación activa y la observación en situaciones reales, unos, y en situaciones simuladas, otros.

\section{REVISIÓN DEL COMPONENTE SOCIOCULTURAL EN ALGUNOS MÉTODOS DE ENSEÑANZA DE UNA LENGUA EXTRANJERA}

El interés por la dimensión cultural del aprendizaje de idiomas no es algo nuevo ni reciente. Aunque lo situamos a partir de la aparición del método comunicativo, encontramos antecedentes en Alemania, Francia, Estados Unidos y en la Europa occidental, pero estas tentativas no llegaron a fraguar en tanto que siempre había existido la tendencia a aislar el aprendizaje de idiomas como sistema gramatical de la provisión de información sobre uno o más países en los que se hablaba ese idioma, continuó, por tanto, siendo el objetivo principal el estudio de la gramática de la lengua extranjera.

Sin embargo, últimamente se hace cada vez más manifiesta la necesidad de ir más allá del mero aprendizaje lingüístico al pretenderse un mayor entendimiento y acercamiento entre culturas encontradas en una situación de aprendizaje de idiomas, ante todo, comunicativo, donde lo importante es la interacción social que desborda los límites del aula (Byram y Fleming, 2001).

A pesar de que durante el s. XIX se hicieron importantes innovaciones metodológicas en el campo de la enseñanza de lenguas extranjeras, prácticamente todas tuvieron como objetivo central la competencia lingüística, sea oral sea escrita, aunque otras veces la naturaleza de la lengua y su aprendizaje tuvieran mayor énfasis.

En el método de Gramática-Tradución (s. XIX) lo importante era "saber todo [los elementos lingüísticos] sobre cualquier cosa más que sobre la cosa en sí [el uso lingüístico]" (W.H.D. Rouse, en Kelly, 1969:53), ello implicaba una visión demasiado academicista de la enseñanza de la lengua. 
A mediados del siglo XIX, entre los movimientos innovadores opuestos a ese método, encontramos las teorías del francés F. Gouin (1831-1896) quien señala la importancia del uso de la lengua y de su contexto para aclarar el significado de los elementos lingüísticos, proponiendo para ello una serie de prácticas que retomarán la Enseñanza Situacional de la Lengua y la Respuesta Física Total. En esos momentos, el énfasis estaba en la competencia oral de la lengua.

A finales del mismo siglo, apareció el Movimiento de Reforma en la enseñanza de lenguas extranjeras (Henry Sweet en Inglaterra, Wilhelm Viëtor en Alemania y Paul Passy en Francia) que revalorizará el estudio de la lengua hablada y de la fonética a través de los métodos naturales, el acento estaba en la lingüística aplicada y su puesta en práctica, se incidía en la importancia del contexto pero aún estaban muy lejos de lo que en estas páginas entendemos por contexto real del uso lingüístico.

Actualmente existen tres tipos de métodos o enfoques generales sobre la naturaleza de la lengua. El primero y más tradicional es el estructural, que considera a la lengua como un sistema de elementos estructurados de manera que codifiquen el significado, métodos en particular (que trabajan desde esta óptica) son el Audiolingüístico y los más modernos la Respuesta Física Total y la Vía Silenciosa.

El segundo enfoque sería de tipo funcional, según el cual la lengua es un medio de expresión de un significado funcional. Esta propuesta se basa en la semántica y la teoría comunicativa, ahora importa el significado y la función, no las estructuras ni la gramática. Aquí incluiríamos el Movimiento Comunitario.

El tercer enfoque general es el interactivo, que considera "la lengua como un vehículo para el desarrollo de relaciones personales y la realización de transacciones de tipo social entre individuos" (Richards y Rodgers, 1998:24). Es ahora cuando se valora el papel de la lengua en las relaciones sociales, los tipos de movimientos, de actos, la negociación y la interacción de los intercambios conversacionales.

Expuestas así estas teorías lingüísticas, consideramos que es la última la que favorece la adquisición de la competencia intercultural de un aprendiz de una lengua no materna. No obstante, los estudiosos de este tipo de enseñanza aseguran que ninguna de las tres son exitosas por sí mismas, sino que podrían complementarse entre ellas.

Seguidamente hagamos una revisión de las ocho propuestas metodológicas de enseñanza de idiomas con más aceptación en las últimas décadas.

- Entre los años 50 y 60 -y aún empleado en los 80-, desde el estructuralismo británico, el Enfoque Oral y la Enseñanza Situacional de la Lengua trabajan a partir de la lengua oral relacionándola con el contexto, la finalidad y la situación real en la que se usa para llegar a una formación de hábitos y de habilidad personal.

El objetivo principal es la enseñanza de una competencia práctica de las cuatro destrezas lingüísticas trabajadas a través de las estructuras y su práctica oral para continuar con la escrita. Se trata de una práctica mecánica y repetitiva en contextos simulados que si bien lleva a la corrección en la producción fonética y estructural, olvida la adecuación real.

- El Método Audiolingüístico parte de los planteamientos de la lingüística estructural americana de los años 50 junto a los postulados psicológicos conductistas de aquella época. Uno de sus principios metodológicos esenciales es el siguiente: 
Los significados que las palabras de la lengua tienen para el hablante nativo pueden aprenderse solamente en un contexto cultural y lingüístico, pero no aisladamente. Por tanto, la enseñanza de la lengua supone la enseñanza de aspectos del sistema cultural que comparten los hablantes de esa lengua.

Pero en la práctica, el centro de atención está en la competencia oral y en la habilidad para responder rápida y correctamente en contextos de comunicación, de nuevo corrección, a partir de ejercicios estructurales y léxicos, y sólo adecuación mencionada en su teoría y se supone que plasmada en algunos aspectos culturales que aparecen en las frecuentes actividades con diálogos a partir de la repetición y memorización de los mismos. Al final, los alumnos eran incapaces de transferir las destrezas desarrolladas a situaciones reales fuera del aula.

- La Enseñanza Comunicativa de la Lengua es un enfoque, con origen británico a finales de los 60, que dio mayor importancia a la competencia comunicativa y a los significados que subyacen en los usos comunicativos de la lengua que al conocimiento de estructuras lingüísticas. El lingüista británico D.A. Wilkins (1972) describe para ello dos tipos de significado: las categorías nocionales (tiempo, secuencia, cantidad, lugar y frecuencia) y las categorías de función comunicativa (pedir, rechazar, ofrecer o quejarse) cuyo papel es tan relevante en cualquier situación comunicativa, las primeras porque a través de esos conceptos reflejan la cultura cotidiana de un pueblo, las segundas porque son necesarias para establecer una comunicación efectiva con personas de ese pueblo.

Respecto a los factores comunicativos y contextuales en el uso de la lengua, se retoman los trabajos del antropólogo Bronislaw Malinowski y del lingüista John Firth, fue este último quien observó que la lengua debía enseñarse en el contexto sociocultural de su uso, incluyendo los participantes, sus conductas y opiniones, los elementos de interacción lingüística y la elección de las palabras (Richards y Rogers, 1998:72).

En un enfoque donde se destaca la práctica como medio para desarrollar las destrezas comunicativas, las funciones lingüísticas principales son la interactiva señalada por Halliday (1973) y la competencia sociolingüística, objeto de nuestro estudio, que es tomada muy en cuenta en este enfoque junto a la estratégica, en términos de Canale y Swain (1980), la primera se refiere a la comprensión del contexto social en que tiene lugar la comunicación y la segunda incluye todas aquellas estrategias que los hablantes emplean para comenzar, mantener, terminar, corregir y reconducir el acto conversacional.

Se trata éste de un enfoque tan amplio que seguidores de otros planteamientos educativos se han visto identificados con él, dando paso a distintas versiones de programas comunicativos: de estructuras y funciones, de espiral funcional alrededor de un eje estructural, estructural-funcional-instrumental, funcional, nocional, interactivo..., muchos de ellos criticados por lo ficticio de sus simulaciones y actividades comunicativas, siendo el interactivo (Widdowson, 1979) y el centrado en tareas (Prabhu, 1983) de los más trabajados en la actualidad.

Entre los tipos de actividades de esta Enseñanza Comunicativa destacan como apropiadas para el desarrollo de la competencia intercultural las de interacción social que incluye conversaciones y discusiones, diálogos e improvisaciones, simulaciones, representaciones y debates. Por otra parte, uno de los ejemplos de materiales de enseñanza empleados son los auténticos o reales, que pueden ser lingüísticos como señales, revistas, anuncios y perió- 
dicos, o gráficos como mapas, dibujos, símbolos, todos ellos para desarrollar actividades comunicativas.

- Un método americano con base en la teoría de la memoria de la Psicología del desarrollo es la Respuesta Física Total, que coordina el habla con la acción y pretende enseñar la lengua a través de la actividad física. En su teoría de aprendizaje James Asher, el autor de este método, hace especial hincapié en el programa biológico innato de aprendizaje de lenguas, en la lateralidad cerebral y las funciones de cada hemisferio para este aprendizaje y en la reducción del estrés. Es un sistema educativo dirigido a un nivel elemental, a los primeros estadios de aprendizaje y es útil como complemento a otros métodos pero no hace referencia ninguna al desarrollo sociocultural y aún menos al intercultural de la lengua meta.

- La Vía Silenciosa, de Caleb Gattegno, se basa en los supuestos del silencio del profesor en el aula y de la animación de este profesor al alumno para emitir el mayor número de enunciados posibles, todo ello apoyado en el uso de regletas y cuadros de colores para la resolución de problemas.

Con un programa estructural y léxico muy tradicional, se centra en el aprendizaje inductivo de la gramática a través de situaciones artificiales representadas por regletas separando la lengua de su contexto social.

Pretende la fluidez, corrección gramatical y de pronunciación como la tiene un nativo, pero no trata la adecuación ni las destrezas de la comunicación.

- En la década de los 70 y los 80 el método Aprendizaje Comunitario de la Lengua de Charles A. Curran se fundamenta en la teoría del Consejo Psicológico, donde el asesorprofesor aconseja y ayuda en sus necesidades al cliente-alumno y donde las prácticas se basan en la alternancia de lengua o el cambio de código.

Entiende la lengua como proceso social, más allá de su concepto como comunicación o mera transferencia de información entre el emisor y el receptor. Dentro del proceso amplio se incluyen otras etapas o subprocesos: el proceso personal, educativo, interpersonal, evolutivo, comunicativo y cultural. De estas dimensiones es la interpersonal, la comunicativa y la cultural las que nos interesan, pues la situación modelo de este método es la interacción doble, entre profesor-alumno y alumno-alumno, no hay éxito en el aprendizaje de la lengua si no se trabajan las relaciones sociales.

De esta forma, el programa, que trata ante todo la lengua oral, no parte de la gramática ni del vocabulario como en otros métodos, sino que "surge de la interacción entre las intenciones comunicativas del alumno y las reformulaciones que hace el profesor para conseguir enunciados adecuados en la lengua objeto" (Richards y Rogers, 1998:117).

Este método pretende alcanzar la adecuación del mensaje por medio de tareas cognitivas, pero sobre todo trabajando la resolución de conflictos afectivos (angustia, ansiedad, inseguridad...) y el respeto a los valores culturales, ya La Forge (1983:66) observa, por ejemplo, cómo las presentaciones personales se hacen de forma diferente según el origen cultural de los alumnos participantes, lo que deben considerarlo ellos mismos para lograr la seguridad cultural en el aprendizaje de la nueva lengua.

- El Enfoque Natural, coetáneo al anterior, fue creado por S. Krashen y T. Terrell y parte de los mismos principios de la enseñanza naturalista de la lengua con los niños pequeños adaptando aquéllos a la adquisición de una segunda lengua. Se trabaja mucho la comprensión antes de llegar a la producción, siempre en situaciones comunicativas y sin recurrir a la lengua materna. 
Considera la lengua como medio de comunicación de significados y mensajes, pero, como en otros muchos métodos, ésta se adquiere mediante un dominio gradual de las estructuras. No obstante, el objetivo principal es la comprensión y expresión adecuada en una situación comunicativa aunque su sintaxis y vocabulario no sean perfectos.

Dentro de la hipótesis de la información de entrada los autores de este método consideran la importancia que para la comprensión tienen la situación y el contexto, la información extralingüística y el conocimiento del mundo que refleja esa información de entrada.

Uno de los papeles fundamentales del profesor es proporcionar un flujo constante y variado de este tipo de información y de elementos extralingüísticos para que los alumnos aprendan a interpretarlos mediante una combinación de actividades de clase que incluyen trabajos grupales, contenidos y contextos múltiples, con materiales que provengan más del mundo real que de los libros de texto.

- El método del psiquiatra búlgaro Georgi Lozanov, la Sugestopedia, aparece al mismo tiempo que los dos anteriores, a finales de los 70, y aún hoy tiene muchos adeptos, sobre todo en la enseñanza no reglada dirigida a directivos y empresarios. Su objetivo es controlar y reconducir las influencias irracionales e inconscientes que reciben continuamente los individuos para mejorar el aprendizaje de una nueva lengua, por ejemplo, trata de manipular la atención para mejorar la memoria por medio de la música.

El alumno trabaja con textos completos e interesantes y audiciones relativas a ellos, además de listas enormes de parejas de palabras, pero la única alusión a la comprensión de contenidos o valores culturales se da en los juegos de roles y en la historia personal nueva que se inventa para cada alumno de acuerdo con la cultura de la nueva lengua.

Tras esta revisión de métodos, a pesar de que muchas de las propuestas mencionan en su programa la importancia de que los alumnos de lengua extranjera alcancen una competencia lingüística, sobre todo, similar a la de un nativo, pocos son los métodos o enfoques de enseñanza que trabajan realmente la competencia intercultural. Podemos destacar la Enseñanza Comunicativa y el Enfoque Natural como los más adecuados para trabajar esta competencia aunque sus procedimientos no llegan a ser revolucionarios.

El peligro del enfoque comunicativo, por ejemplo, radica en que la lengua que se trabaja en el aula puede ser muy diferente a la empleada en una situación comunicativa real, un estudio de Long y Sato (1984) (en Richards y Rodgers, 1998:156-157) comprobó que en muchas de sus actividades el uso lingüístico era parecido al de los ejercicios mecánicos de preguntas y respuestas planteados por el Método estructuralista Audiolingüístico.

\section{Conclusión}

Cuando aprendemos una lengua, nuestra meta es, ante todo, lograr lo que nos proponemos al usar el lenguaje, evitando malentendidos y decidiendo esos usos en función de nuestros propósitos, esto no se alcanza más que interviniendo en distintas situaciones, unas simuladas, otras reales, y analizando y evaluando nuestra participación en relación con la de los demás.

Como profesores, debemos esforzarnos en formar usuarios cada vez más competentes de las lenguas o, lo que es lo mismo, desarrollar en nuestros alumnos la capacidad de producir e interpretar lo que se dice de acuerdo con un contexto y de adaptar los usos de la lengua 
con diferentes propósitos. Unimos pues formas lingüísticas con conocimiento del mundo los savoirs que incluye Byram (1995) en la competencia intercultural-.

Ante este nuevo objetivo que se propondrá, si no lo hizo antes, el profesor de una L2 o una lengua extranjera, debe replantear su programación y diseñar nuevas actividades y recursos que se orienten eficazmente a la consecución de la competencia intercultural, rehaciendo un análisis de los usos lingüísticos y comunicativos no hacia atrás, hacia lo hecho, sino de cara a lo que ha de venir, al mundo que se abre ante el alumno de la nueva lengua, aunando al mismo tiempo conocimiento de formas lingüísticas y su uso y adecuación al contexto, procurando estrategias que lo ayuden a superar problemas de comunicación.

Es importante, como señala Unamuno (2003:125), poner a disposición de quienes aprenden no sólo situaciones y usos comunicativos (orales y escritos) que actúen como modelos, sino herramientas de análisis de dichas situaciones y usos, lo que les permitiría ir adquiriendo autonomía en la adecuación lingüística que exige su participación social.

\section{REFERENCias Bibliográficas}

Adam, J.M. (1985). "Quels types de textes" en Le Français dans le Monde, 192.

Baliga, C. Y Baker, J. (1985). "Multinational corporate policies for expatriate managers: selection, training and evaluation", SAM Advanced Management Journal, Vol. 50, 4.

Brewster, C. (1991). The management of expatriates. Cranfield: Cranfield Institute of Technology. Human Resource Centre Monograph 2.

Byram, M. (1995). "Acquiring Intercultural Competence. A Review of Learning Theories" en L. Sercu (ed.), Intercultural Competence. 1: 53-70.

Byram, M. y Fleming, M. (2001). Perspectivas interculturales en el aprendizaje de idiomas. Enfoques a través del teatro y la etnografía. Cambridge: Cambridge University Press.

Cambra, M. (1989). "La compréhension des textes écrits en français langue étrangère à EGB" en Qüestions obertes de didáctica de la Llengua. Universitat de Barcelona, 63-76.

Canale, M. y Swain, M. (1980). "Theoretical bases of communicative approaches to second language teaching and testing" en Applied Linguistics, 1 (1), 1-47.

Chen, G.-M.(1998). "Intercultural communication via e-mail debate" en The edge: The E-Journal of Intercultural Relations, 1 (4), pp. 1-14.

Favaro, G. (2000). "Accogliere, comunicare, scambiare. Costruire progetti interculturali tra scuola e territorio" en I Centri Interculturali: mappa, azioni, parole chiave (Materiali del Secondo Incontro Nazionale, Venecia, 27 e 28 ottobre 1999). Venecia: Servizi Educativi, Centro Documentazione Educativa, 21-41.

Hains, A.H., Lynch, E.W. y Winton, P.J. (1997). Cultural Competence: a Review of the Literature. Unpublished manuscript, CLAS Early Childhood Research Institute, Champaing, IL.

Hall, E.T. (1978). Más allá de la cultura. Barcelona: Gustavo Gili.

Hall, E.T. (1986). La dimensión oculta. México: Siglo Veintiuno Editores.

Hall, E.T. (1989). El lenguaje silencioso. Madrid: Alianza Editorial.

Halliday, M.A.K. (1973). Explorations in the Functions of Language. Londres: Edward Arnold. Hofstede, G. (1984). Culture's Consequences. London: Sage.

Kelly, L.G. (1969). 25 Centuries of Language Teaching. Rowley, Massachusetts: Newbury House. 
La Forge, P.G. (1983). Counseling and Culture in Second Language Acquisition. Oxford: Pergamon. M.E.C.D. (2003). REAL DECRETO 830/2003, de 27 de junio, por el que se establecen las enseñanzas comunes de la Educación Primaria. Madrid: B.O.E. n. ${ }^{\circ}$ 157: 25460-25462.

M.E.C.D. (2003). REAL DECRETO 831/2003, de 27 de junio, por el que se establece la ordenación general y las enseñanzas comunes de la Educación Secundaria Obligatoria. Madrid: B.O.E. n. ${ }^{\circ}$ 158: 25694-25706.

Meyer, M. (1991). "Developing Transcultural Competence: Case Studies of Advanced Foreing Language Learners" en D. Butjes y M. Byram (eds.): Developing Languages and Cultures.

Oliveras, A. (2000). Hacia la competencia intercultural en el aprendizaje de una lengua extranjera. Estudio de choque cultural y los malentendidos. Madrid: Edinumen.

Porter, D. y Roberts, J. (1981). « Authentic listening activities » eEnglish Language Teaching Journal, 36, 1: 37-47.

Prabhu, N. (1983). "Procedural syllabuses". Ponencia presentada en RELC Seminar, Singapur.

Richards, J.C. y Rodgers, T.S. (1998). Enfoques y métodos en la enseñanza de idiomas. Cambridge: University Press.

Rivers, W.M. (1964). The Psychologist and the Foreign Language Teacher. Chicago: University of Chicago Press.

Surian, A. (2000). "Educare in prospettiva interculturale" en I Centri Interculturali: mappa, azioni, parole chiave (Materiali del Secondo Incontro Nazionale, Venecia, 27 e 28 ottobre 1999). Venecia: Servizi Educativi, Centro Documentazione Educativa, 43-50.

Trujillo Sáez, F. (2001). “Objetivos en la enseñanza de lenguas extranjeras: de la competencia lingüística a la competencia intercultural, en F. Herrera Clavero, F. Mateos Claros, S. Ramírez Fernández, M. I. Ramírez Salguero y J. M. Roa Venegas (coords.) Inmigración, interculturalidad y convivencia. Ceuta: Instituto de Estudios Ceutíes, pp. 407-418. Disponible en http://www.ugr.es/ ftsaez/research.htm.

Unamuno, V. (2003). Lengua, escuela y diversidad sociocultural. Hacia una educación lingüística crítica. Barcelona: Graó.

Van Ek, J. (1984). Across the threshold readings from the modern languages projects of the Council of Europe. Oxford: Pergamon Press.

Vilà, R. (2002). "El desarrollo de la competencia comunicativa intercultural en una sociedad multicultural y plurilingüe: Una propuesta de instrumentos para su evaluación". (Comunicación presentada en el XXIII Seminario de Lenguas y Educación: Ensenyar llengües en la diversitat $i$ per a la diversitat (30 de mayo al 1 de junio 2002, Barcelona). Disponible en el espacio de Internet: Portaling: Una porta oberta a les llengües.

Widdowson, H.G. (1979). "The communicative approach and its applications" en H.G. Widdowson. Explorations in Applied Linguistics. Oxford: Oxford University Press.

Wilkins, D.A. (1972). The Linguistic and situational content of the common core in a unit/credit system. Estrasburgo: Consejo de Europa. 